\title{
Erratum: Condensation, demixing, and orientational ordering of magnetic colloidal suspensions [Phys. Rev. E 91, 052127 (2015)]
}

\author{
Stefanie M. Cattes, ${ }^{*}$ Sabine H. L. Klapp, and Martin Schoen
}

(Received 24 October 2018; published 12 November 2018)

DOI: 10.1103/PhysRevE.98.059901

In the original paper we argue that, because of our choice of $\varepsilon_{11}=\varepsilon_{22}$ (considering only binary pseudomixtures), $u_{0}^{(11)}=u_{0}^{(22)}$ in Eq. (3.9) of the original paper. This statement is incorrect because the energy parameters $u_{l}^{(a b)}$ are proportional to the expansion coefficients $f_{l l 0}^{(a b)}$ [see Eq. (3.9) of our original paper]. In particular, for $l=0$ and $a=b=2$, the latter are given by (see the Appendix of Ref. [1]) $f_{000}^{(22)} /(4 \pi)^{3 / 2} \propto 1+\varepsilon_{\mathrm{H}}^{2}$ where $\varepsilon_{\mathrm{H}}$ is a coupling constant that determines the interaction strength between a pair of spins of molecules of component 2 . The correction proportional to $\varepsilon_{\mathrm{H}}$ is missing in the corresponding expression for $f_{000}^{(11)}$ because particles of component 1 in our model do not carry a spin and therefore $f_{000}^{(11)} \neq f_{000}^{(22)}$.

However, we stress that because the largest coupling constant used in the original paper is $\varepsilon_{\mathrm{H}}=0.12$ and because $f_{000}^{(22)} /(4 \pi)^{3 / 2} \propto 1+\varepsilon_{\mathrm{H}}^{2}$ the error introduced by erroneously assuming that $f_{000}^{(11)}=f_{000}^{(22)}$ is vanishingly small and causes only a minute shift of the entire phase diagram to lower temperatures $T$. This shift is altogether inconsequential for the overall topology of the phase diagram at a given concentration of the magnetic colloids.

To correct the error in the equations we presented in the original paper we need to replace $u_{0}$ by $u_{0}^{(a b)}$ where $a=b=1$ in Eq. (3.19) [see also Eq. (A3)] and $a=b=2$ in Eqs. (3.20) and (3.23) [see also Eq. (A2)] of our paper. Similarly, in Eqs. (3.24) and (A1) of the original paper, $u_{0}\left(\rho_{1}^{2}+\rho_{2}^{2}\right) \rightarrow u_{0}^{(11)} \rho_{1}^{2}+u_{0}^{(22)} \rho_{2}^{2}$.

Moreover, from Eq. (3.18) of our paper it can be realized that the (normalized) orientation distribution function can be cast as

$$
\bar{\alpha}(x)=\frac{\Psi(x ; H)}{\int_{-1}^{1} d x \Psi(x ; H)},
$$

where $\Psi$ is given in Eq. (3.22) of the original paper. Thus, the correct expression replacing Eq. (3.21) of our paper then becomes

$$
\int_{-1}^{1} d x \bar{\alpha}(x) \ln [2 \bar{\alpha}(x)]=-\ln \left[\frac{1}{2} \int_{-1}^{1} d x \Psi(x ; H)\right]-2 \rho_{2} \sum_{l=1}^{\infty} u_{l} \alpha_{l}^{2}+\beta H \mathcal{P}_{1} .
$$

Comparing Eq. (2) with Eq. (3.21) of the original paper one realizes that the first term appearing on the right hand side of the latter should not arise whereas the last term in Eq. (2) is missing. As a consequence, the third term on the right hand side of Eq. (3.23) of our original paper should not appear. However, we stress that all the numerical results presented in the original paper are based on the correct expressions and therefore remain valid despite the typographical errors in the paper.

Last, but not least, the terms proportional to $2 \eta^{\prime}$ and $2 \eta^{\prime \prime}$ should be replaced by $2 \eta^{\prime 2}$ and $2 \eta^{\prime \prime 2}$ in Eq. (A1) of the original paper. Again, this is only a typographical error in the paper not affecting any of the results presented earlier in our paper.

[1] S. M. Wandrei, D. G. McCarthy, and M. Schoen, Langmuir 33, 11366 (2017).

*Current author name: Stefanie M. Wandrei. 